\title{
Perspective
}

PERSPECTIVE Actualité en histoire de l'art

$1 \mid 2020$

Japon

\section{Gutai en tension : pour une histoire systémique des arts au stade de la mondialisation}

Gutai Under Strain: Toward a Systemic History of the Arts in a Globalized World Gutai im Spannungsfeld einer systemischen Geschichte der Künste im Zeitalter der Globalisierung

Gutai in tensione: per una storia sistemica delle arti al tempo della globalizzazione

Gutai en tensión: por una historia sistemática de las artes en el estadio de la mundialización

\section{Michael Lucken}

\section{OpenEdition}

Journals

Édition électronique

URL : http://journals.openedition.org/perspective/19272

DOI : 10.4000/perspective. 19272

ISSN : 2269-7721

\section{Éditeur}

Institut national d'histoire de l'art

\section{Édition imprimée}

Date de publication : 5 juin 2020

Pagination : 281-292

ISBN : 978-2-917902-89-9

ISSN : $1777-7852$

\section{Référence électronique}

Michael Lucken, «Gutai en tension : pour une histoire systémique des arts au stade de la mondialisation », Perspective [En ligne], 1 | 2020, mis en ligne le 30 décembre 2020, consulté le 25 janvier 2021. URL : http://journals.openedition.org/perspective/19272 ; DOI : https://doi.org/10.4000/ perspective. 19272 


\title{
Gutai en tension : pour une histoire systémique des arts au stade de la mondialisation
}

\author{
Michael Lucken
}

En 1957, le critique Hariu Ichirō écrit en réaction aux premiers succès remportés par le mouvement Gutai (1954-1972) sur la scène artistique japonaise :

Derrière l'Informel, il y a la tradition de l'humanisme européen fondée sur la dynamique du renversement, et c'est ce que les artistes occidentaux cherchent au travers de l'exploration de la matière. C'est en cela qu'ils s'opposent à la réalité actuelle. Mais où sont ces objectifs contestataires dans le groupe Gutai ? Il est très bien que chacun ait le droit de s'exprimer librement sur la scène internationale, mais il faudrait aussi être clair sur le fait que ce qui relève de la contestation en Europe ne peut pas devenir, tel quel, contestataire au Japon ${ }^{1}$.

Cette observation pose une question de fond qui dépasse le seul cas japonais. Dès lors qu'elles ont été produites dans des contextes socioculturels distincts, les formes ont beau être similaires, elles ne coïncident pas. L'« abstraction » dans un pays est-asiatique qui possède une longue tradition critique d'appréciation de la ligne, du geste et de la trace peut difficilement être jugée de façon pertinente suivant les critères de l'histoire occidentale. Ce phénomène est d'autant plus marqué que les espaces sont interconnectés, car cela introduit des stratégies d'imitation, de réaction et de repositionnement qui, par nature, suscitent écarts et distorsions. Il faut donc tenir compte d'une double dynamique de différentiation, l'une de nature diachronique, l'autre de nature synchronique. Pour le dire autrement, l'aspect, la texture, le style des œuvres peuvent se ressembler, mais leur sens est différent. Il existe un phénomène de pseudomorphisme qu'il est urgent de remettre en évidence.

Le pseudomorphisme est un concept difficile à manier, car défaillant sur ses deux bords. Pris de manière trop abstraite, il suppose l'existence en miroir d'une vérité de la forme - celle du référent - qui n'est guère satisfaisante. Pris de manière trop étroite, il ne renvoie qu'à la variété infinie des combinaisons élémentaires et au constat peu opératoire qu'au niveau micro les formes produites sont « semblables et pourtant dissemblables ${ }^{2}$ ». $\mathrm{C}^{\prime}$ est la raison pour laquelle il n'est vraiment pertinent que pour décrire des dynamiques collectives et sociales. En l'occurrence, nous l'utiliserons dans une perspective nationale, car c'est surtout à ce niveau que s'est jouée la réception de Gutai. Le pseudomorphisme 
n'est donc pas seulement une question de forme : sous les aspects formels se cachent des questions d'ordre moral et politique, des questions de prééminence symbolique, de rapports de forces, qui rejouent sur le plan artistique une histoire complexe entre les peuples, faite de domination et de fascination, d'échanges et de compénétrations. Rendre compte et analyser le fonctionnement de ce mécanisme à travers le prisme du mouvement Gutai a pour objectif de contribuer à la réflexion en cours sur les conditions de possibilité d'une histoire systémique des arts au stade de la mondialisation.

\section{Réceptions croisées}

Depuis la visite qu'a rendue le critique d'art Michel Tapié aux artistes de Gutai à Osaka en 1957, la France, avec l'Italie, a toujours joué un rôle de premier plan dans la réception internationale du mouvement japonais. Plusieurs auteurs importants, à commencer par Alfred Pacquement, Laurence Bertrand Dorléac et Marc Dachy, ont écrit sur le groupe japonais ${ }^{3}$. Aucun ne remet en cause son importance, le débat ayant comme principal foyer la contribution de Tapié, positive pour certains, néfaste pour d'autres ${ }^{4}$. La seule exception notable est Georges Mathieu, dont le jugement est lapidaire, mais la rivalité dans son cas est trop directe pour que son point de vue compte véritablement. L'impression qui domine est que Gutai, à travers la figure de Tapié, fait partie d'une histoire nationale et, plus généralement, que la France comprend Gutai - du moins les réalisations des années 1950 -, mieux que les États-Unis, où le groupe a longtemps été ignoré, mieux même que le milieu de l'art japonais, dont l'hostilité est de notoriété publique. Pris de manière collective, cet accueil favorable apparaît comme une manière de " redonner à la nation une dimension mondiale ", ainsi que le souligne Alexandra Munroes.

Les États-Unis, pour leur part, ont longuement hésité à accorder de l'intérêt au groupe piloté par Yoshihara Jirō. Les réactions furent d'emblée circonspectes. Dore Ashton par exemple relève en 1958 l'influence de Jackson Pollock sur Gutai et, non sans préjugés orientalistes, insiste sur le caractère immature et irréfléchi des œuvres produites par le groupe . $^{6}$ Michael Kirby a certes fait remarquer quelques années plus tard la dimension pionnière des performances de Gutai, mais cette reconnaissance dans les milieux underground est restée un succès d'estime ; elle n'a entraîné aucune publication, ni exposition significatives, comme si les critiques américains redoutaient au fond l'irruption d'un autre rival que la création européenne et française en particulier ${ }^{7}$. Pour le dire autrement, le cadre hégémonique des stratégies nationales détermine de manière fondamentale l'appréciation de Gutai en Occident, la France jouant la carte du primus inter pares face à l'impérialisme américain.

Une évolution a toutefois vu le jour aux États-Unis dans les années 1990 grâce aux efforts de spécialistes comme Reiko Tomii, Alexandra Munroe et Ming Tiampo. Mais ce n'est que récemment qu'un changement s'est opéré à plus grande échelle, surtout depuis 2013 et la rétrospective que ces deux dernières ont organisée au musée Guggenheim à New York. Gutai fait désormais l'objet d'une critique qui se veut transnationale, qui est sensible à toutes les différences individuelles, qui recherche le décentrement, les contacts et les connexions, mais qui ne circule quasiment qu'en anglais, et dont l'intérêt pour les échanges et la prétention à la globalité sont trop en osmose avec l'ordre économique américain pour qu'on puisse la considérer comme affranchie des intérêts locaux ${ }^{8}$.

Après cet exposé succinct des positions occidentales, essayons de comprendre la nature du " problème Gutai » dans l'archipel. Bien que quelques conservateurs et universitaires aient passionnément soutenu le groupe, à commencer par Kawasaki Kōichi, Osaki Shin'ichirō et Hirai Shōichi' ${ }^{9}$, bien qu'ils aient publié des ouvrages essentiels, comme les Documents Gutai en 1993, ou organisé de grandes rétrospectives, comme celle qui s'est 
tenue en 2012 au Nouveau Centre national des beaux-arts (Kokuritsu shin-bijutsukan), l'imprégnation du mouvement dans le tissu de la critique contemporaine est ambiguë. Le fait que des artistes aussi importants que Shiraga Kazuo et Shimamoto Shōzō n'aient pour l'heure fait l'objet d'aucune monographie critique en japonais en est un premier signe. Il paraît impossible d'affirmer, comme le fait Ming Tiampo, que "l'héritage de Gutai au Japon est d'une importance quasi ineffable ${ }^{10} »$. Ou alors sur un mode beaucoup plus secret, tourmenté et conflictuel qu'elle ne le perçoit.

La méfiance avec laquelle Gutai a été reçu dans le milieu de l'art japonais n'est pas un mystère ${ }^{11}$. Plusieurs auteurs ont tenté d'expliquer au public occidental les raisons de ce "désaveu systématique ", à l'instar d'Okabe Aomi dans le catalogue de l'exposition Le Japon des avant-gardes ${ }^{12}$, ou d'Itō Junji dans le catalogue de la rétrospective du musée du Jeu de Paume en $1999^{13}$. A notamment été mise en avant la méfiance de Tōkyō visà-vis du Kansai où le mouvement s'est développé. Mais ce prisme de lecture «province contre capitale " est fragile car il renvoie à la posture même du groupe à l'époque. Aussi tenterons-nous de rendre compte d'autres aspects de la réception japonaise de Gutai, en commençant par le monde académique.

L'Université japonaise n'a produit qu'un nombre très restreint de travaux sur Gutai, la seule thèse soutenue étant celle de Hirai en $2006^{14}$. Il y a certes là un phénomène plus large qui veut qu'il y ait beaucoup moins de thèses en histoire de l'art au Japon qu'en France, et que la production artistique post-1945 en particulier soit encore très peu étudiée, toutefois on peut se demander dans quelle mesure l'occultation de ce que l'historiographie japonaise appelle le " contemporain " (gendai) n'a pas un lien avec la disparité qui existe en Occident entre l'assimilation rapide de certaines œuvres d'après-guerre - à commencer par celles de Gutai - et l'absence de tout intérêt pour la période considérée comme " moderne " ou kindai en japonais, située entre 1868 environ et 1945. Tant que la production japonaise " moderne " ne sera pas acceptée sur la scène internationale, le « contemporain " restera problématique. Il est ainsi frappant et révélateur qu'aucun des grands spécialistes du domaine n'ait vraiment franchi le Rubicon de 1945. Qu'il s'agisse de Tan'o Yasunori, Omuka Toshiharu, Kinoshita Naoyuki, Ushiroshōji Masahiro, Satō Dōshin ou Inaga Shigemi, tous sont restés centrés sur les périodes Meiji (1868-1912), Taishō (1912-1926) et début Shōwa (1926-1945) et, malgré leur grande curiosité d'esprit, ils n'ont encouragé les travaux sur les périodes suivantes qu'à la marge ${ }^{15}$. Depuis les années 1980-1990 et l'émergence de l'histoire de l'art moderne comme champ de recherche à part entière, Gutai a, dans l'ensemble, été traité par les chercheurs avec une forme d'hostilité larvée ${ }^{16}$. La situation pourrait changer avec le renouvellement de génération en cours, mais il est encore trop tôt pour se prononcer ${ }^{17}$. Notons cependant le développement d'études portant sur les femmes du mouvement, comme Tanaka Atsuko, indice d'une réévaluation par le biais du genre d'un groupe tenu à distance par un monde académique centré à Tōkyō et massivement dominé par les hommes ${ }^{18}$.

Hors du champ universitaire, la situation n'est guère plus favorable, mais les arguments sont plus explicites. Dans L'anarchie des corps, ouvrage de référence publié par le critique et conservateur Kuroda Raiji en 2010 sur les mouvements underground des années 1960 où l'on s'attendrait, d'un point de vue occidental, à ce que le groupe Gutai occupe une place éminente, ce dernier n'est que brièvement présenté et la conclusion est lapidaire :

On a gardé des images couleurs du spectacle proposé pour l'exposition universelle [d'Ōsaka] par Gutai, [collectif] qui réalisa en avance sur le monde entier des actions sur le mode de la performance : un show niais et familial, qui n'est pas sans rappeler la nullité et l'impuissance de "l'art" actuel - ainsi que l'indigence de l'histoire de l'art depuis Gutai ${ }^{19}$. 
L'idée que Gutai fut un mouvement superficiel et commercial est l'un des traits qui ressort le plus, en particulier chez les auteurs marqués à gauche, comme c'est ici le cas. La dette du groupe à l'égard de Pollock et de l'expressionnisme abstrait, son absence de tout engagement politique dans les années 1950, son refus d'emboîter le pas de la contestation sociale dans la décennie suivante, le confort matériel dont il a bénéficié grâce à la fortune de Yoshihara Jirō, situent Gutai comme un mouvement d'avant-garde bourgeois et, même si l'emprise du marxisme est aujourd'hui beaucoup moins forte qu'elle ne l'a été jusque dans les années 1990, le sentiment diffus que le collectif d'Ashiya représente les intérêts d'une classe dirigeante et américanisée n'a pas disparu.

Toutefois, Gutai réunit aussi contre lui de nombreux auteurs sensibles aux questions postcoloniales et identitaires. L'échec de Gutai ne tient pas pour eux à son absence de conscience politique, à sa défense de "l'art pour l'art ", mais à son opportunisme, à son incapacité à résister aux sirènes du succès et à s'imposer comme le porte-drapeau de la nouvelle créativité nippone. Dans cette perspective, le nœud du problème est l'attitude du groupe vis-à-vis de Tapié et des exigences du marché européen, le fait que les performances aient été reléguées au second plan après 1960 au profit d'une peinture calibrée. Gutai s'est non seulement trahi lui-même, mais il a trahi tout un pan de l'histoire nationale. Ce qui ressort en effet de cet épisode, c'est qu'au moment même où, pour la première fois, un mouvement artistique japonais s'est imposé en temps réel sur la scène internationale, concrétisant ainsi les espoirs de générations d'artistes depuis la fin du XIX ${ }^{e}$ siècle, il a aussitôt baissé pavillon et accepté la domination du point de vue européen. " En d'autres termes, un mouvement artistique indépendant, né au Japon dans l'idée de ne jamais imiter personne" et qui a démarré sans aucun lien avec l'informel, s'est retrouvé confiné dans une position subalterne et mimétique vis-à-vis du centre occidental ", écrit de façon limpide l'artiste Nakazawa Hideki ${ }^{20}$.

À ce reproche, s'ajoute volontiers une critique de fond sur le sens de l'action et de l'engagement au Japon. Sawaragi Noi est certainement celui qui a développé cette ligne d'attaque de la manière la plus forte et structurée ${ }^{21}$. Dans Art / Japon / Contemporain (1998), ouvrage qui a rencontré un écho considérable et porté sur le devant de la scène une nouvelle génération d'artistes (Murakami Takashi, Aida Makoto...), Sawaragi part du constat que l'art japonais " moderne " et " contemporain » en général n'est jamais parvenu à s'affranchir d'une relation à l'Occident de type colonial. Gutai possède cependant une place à part dans son pandémonium. L'argumentation est la suivante. Là où les artistes occidentaux ont vu dans l'expressionnisme abstrait une possibilité de conquête qui était autant celle, formelle, de l'espace de la toile, que celle, politique, des ateliers du monde entier, quand s'y découvraient donc pour eux un futur excitant et des libertés nouvelles, au Japon, dans un contexte où personne n'avait oublié les conditions de la fin de la guerre (gestes héroïques désespérés, engloutissement de la volonté individuelle dans le collectif...), Gutai n'y a trouvé qu'un retour à la matière, à la praxis, impliquant du même coup un retrait du politique et l'abandon de toute poièsis, entendue comme la capacité de l'homme à transformer le monde conformément à ses idées ${ }^{22}$. Gutai caractérise pour Sawaragi le moment où les artistes japonais, au lieu de chercher à reconquérir la véritable autonomie de projet que devait leur permettre la fin de l'occupation américaine, s'installent vis-à-vis de l'Occident dans une posture de repli dans l'action immédiate et la matière brute, posture de défaite dont il perçoit les rebonds dans tous les mouvements anti-art (han-geijutsu) des années 1960 et jusqu'à Mono-ha dans les années $1970^{23}$. À charge donc pour la nouvelle génération d'artistes

1. Murakami Saburō, Passage (Kami-yaburi), performance, 1955-1956. 


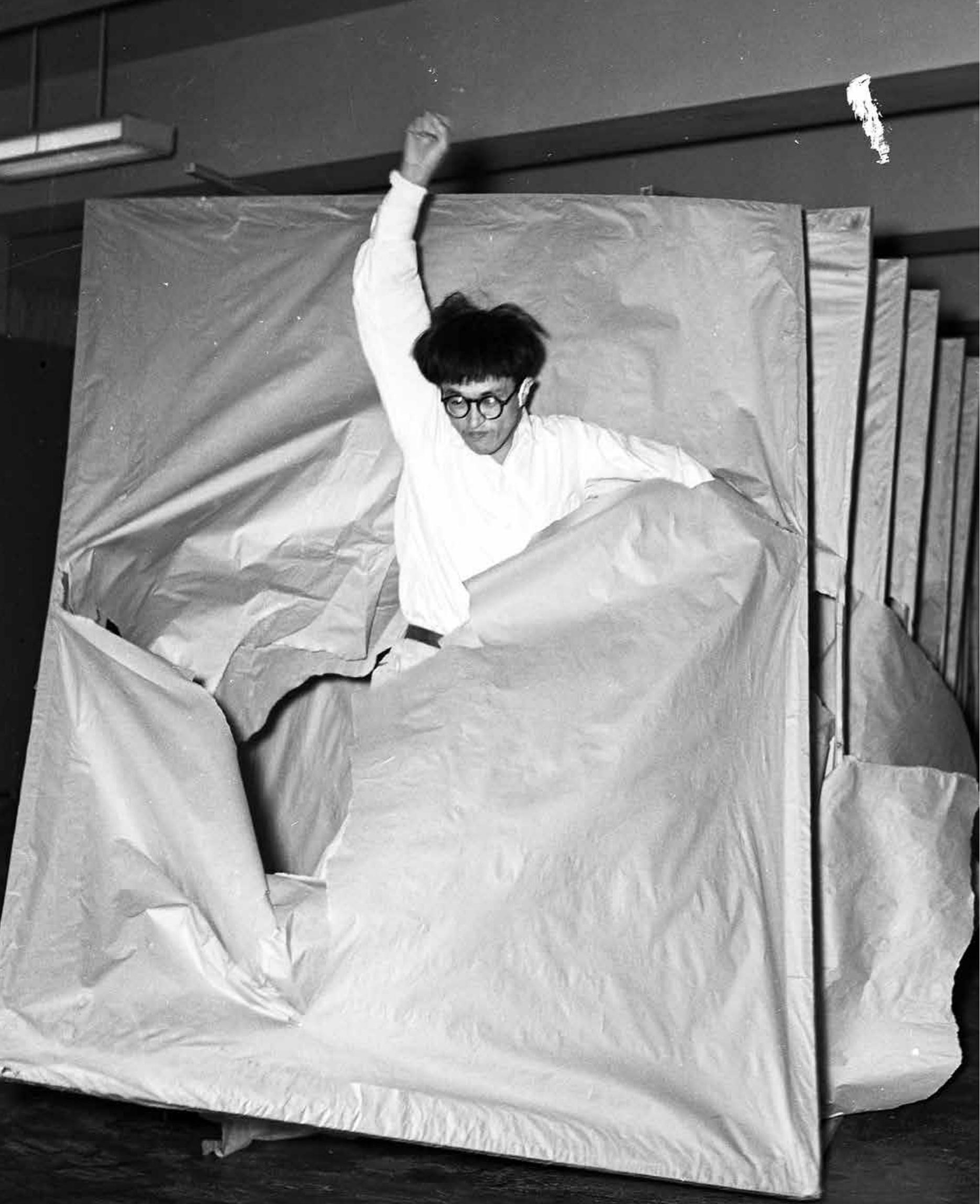




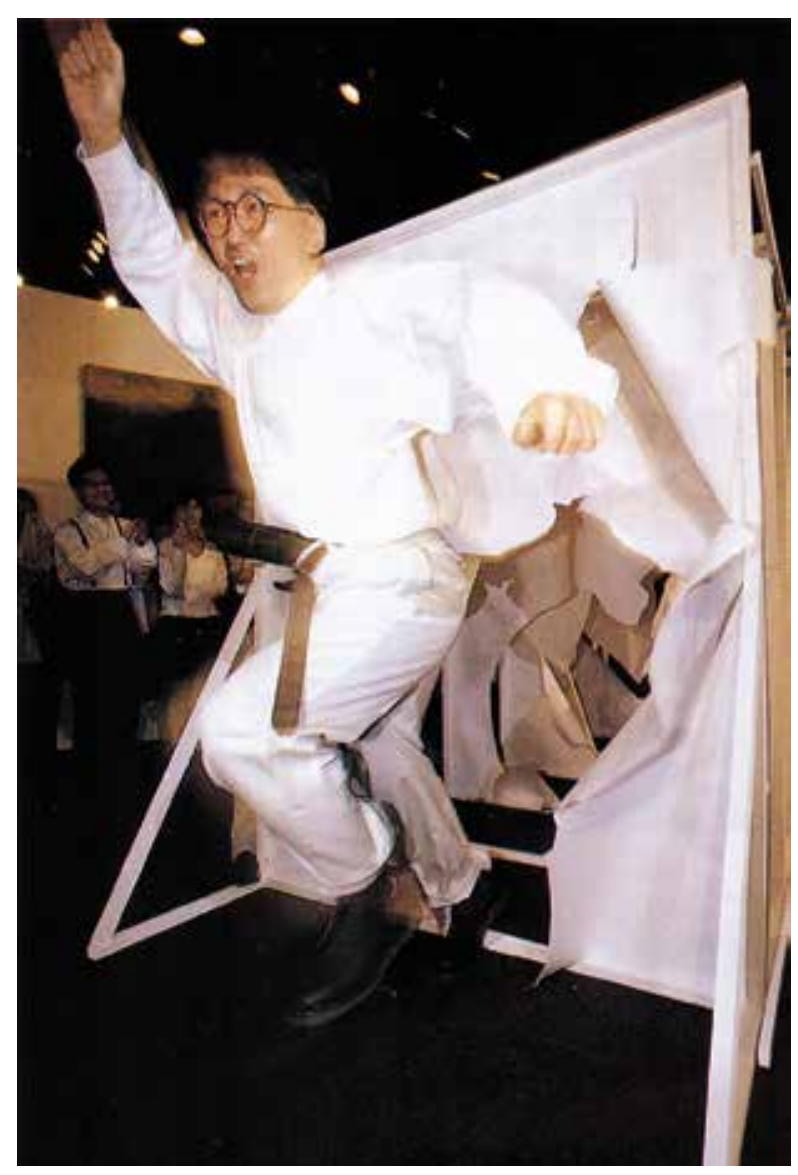

2. Murakami Takashi, Passage (Kami-yaburi), performance, 1992 (photographie de Kurokawa Mikio). qu'il défend d'essayer de sortir de la logique $<$ projet occidental / neutralisation japonaise (au sens électrique d'un passage à la terre) $>$, telle qu'elle s'est installée autour de 1955 et les premières grandes manifestations de Gutai $^{24}$.

La reprise parodique par Murakami Takashi (1992) de la performance de son homonyme de Gutai Murakami Saburō (1955) traduit ce point de vue sous une forme plastique (fig. $\mathbf{1}$ et $\mathbf{2}$ ). Alors qu'on pourrait avoir tendance en Occident à voir dans le passage impétueux de Murakami Saburō à travers des écrans de papier un symbole de liberté triomphante, presque une résurrection du chef-d'œuvre de Delacroix, Murakami Takashi, en reproduisant le geste de son aîné sur un mode burlesque, propose une interprétation tout autre. Les écrans de papier alignés les uns derrière les autres deviennent la métaphore des modes occidentales face à la succession desquelles l'artiste « japonais » est laissé avec uniquement deux options : réagir en copiant ou, comme le fait l'artiste de Gutai, tenter vainement de passer de l'autre côté du mur au détriment de tout projet personnel ${ }^{25}$. Se dessine en clair-obscur une histoire japonaise réduite soit à l'ancillarité et la subséquence, soit à la brutalité et la saisie absurde du réel. Le personnage de peintre qu'incarne Kitano Takeshi dans son film Achille et la tortue (2008) représente parfaitement cette double impasse. Le fait que Murakami Takashi ait conçu toute son ouvre autour de la notion de "stratégie " (senryaku) - qu'elle soit artistique, culturelle, médiatique ou marchande - est la conséquence directe de cette lecture d'un art japonais d' « après-guerre » ayant échoué à se projeter par l'esprit dans le futur et à rayonner de façon autonome.

Il existe donc un hiatus entre les différents modes de réception de Gutai. Enthousiaste en Europe de l'Ouest ; circonspect et distant aux États-Unis - du moins jusqu'à tout récemment ; ambivalent au Japon, entre soutien actif d'un petit nombre et rejet par tous les autres. Mais, au-delà, ce qui ressort est le manque d'attention pour les formes. À l'instar de ce que montre Béatrice Joyeux-Prunel à propos des avant-gardes occidentales, les différentes réceptions de Gutai sont avant tout déterminées par des considérations d'ordre national et géopolitique ${ }^{26}$. Le jugement de goût, l'appréciation esthétique, au sens d'un ressenti sensible, donnent le sentiment de n'avoir qu'une pertinence faible à un niveau macro-historique. Or, si le politique domine dans une perspective transnationale, l'analyse des formes doit nécessairement en conserver la trace lorsqu'on se situe au niveau des réalisations plastiques. Comment intégrer dans la pensée des ouvres les rapports de force et la différenciation? 


\section{Pseudomorphisme}

Analyser un panneau de faux marbre peint par Fra Angelico à l'aune des drippings de Pollock : telle est la proposition « anachronique » faite par Georges Didi-Huberman en ouverture de Devant le temps. Ce questionnement présente des similitudes avec le nôtre, mais aussi des différences éclairantes.

Prenons les tableaux peints au pied par Shiraga. D'une part, il paraît évident qu'ils s'inscrivent dans une tradition qui valorise le geste, la trace, la texture, le travail à plat et, donc, une forme d'engloutissement de l'artiste dans l'œuvre, à la manière de Hokusai obligé de marcher sur sa feuille de papier pour peindre un immense portrait à l'encre de Daruma. De l'autre, une forme d'instinct analogique pousse le spectateur à les rapprocher de l'abstraction lyrique européenne et de l'expressionnisme abstrait américain, c'est-à-dire des œuvres créées dans un tout autre contexte. Dans les deux cas, qu'il s'agisse de Fra Angelico ou de Shiraga, on peut dire avec Didi-Huberman que " la ressemblance ressortit au domaine de ce qu'on appelle un pseudomorphisme ${ }^{27}$ ".

Les formes sont similaires, mais les trajectoires sont différentes. Depuis Panofsky, la métaphore géologique de la pseudomorphose a souvent été utilisée en histoire de l'art. Mais comme le montre Andrea Pinotti, elle est presque toujours pensée comme un problème qu'il convient soit de réduire, soit d'écarter, dans le prolongement de la critique platonicienne de la mimèsis ${ }^{28}$. Or le raisonnement qu'applique Didi-Huberman à la temporalité des œuvres pourrait s'appliquer pareillement au niveau spatial. En effet, s'il existe une "fatalité de l'anachronisme ${ }^{29}$ ", on peut parler également d'une fatalité $d u$ pseudomorphisme qui, par bien des aspects, est à l'espace ce que le premier est au temps, à savoir une perturbation de la continuité. Bien que produites à la même époque, les œuvres de Shiraga ne sauraient être assimilées à celles de Georges Mathieu et Franz Kline. Il y a toujours quelque chose qui résiste, gêne la superposition et rend fragile les catégories.

Depuis Bergson et Heidegger, l'idée d'une pluralité des temps humains est couramment acceptée par la philosophie occidentale. En revanche, concevoir l'espace sous l'angle de la pluralité semble encore poser des difficultés. Didi-Huberman par exemple est très sensible aux anachronismes et à l'archéologie des formes, mais les espaces qu'il interroge sont, dans l'ensemble, lisses et homogènes. La Renaissance florentine n'est pas un Ailleurs, au contraire, quasiment rien ne lui semble " aussi connu, aussi bien "documenté" 30 ". De même, lorsqu'il s'intéresse à Aby Warburg, aux photographies des camps d'extermination ou à la théorie du cinéma d'Eisenstein : qu'ils soient " italiens ", " allemands" ou " soviétiques ", les milieux humains présentent peu d'aspérités, les langues sont perméables, et la pensée les traverse sans heurt. Le temps et les formes sont dans un rapport complexe et tendu, mais l'espace - géographique, culturel, linguistique, scriptural - fournit une solution qui permet de (re-)donner cohérence à l'ensemble. La proposition que je formule est que le pseudomorphisme n'est un obstacle que dans le cadre d'une vision unifiée et autocentrée du monde. Dès qu'on s'en éloigne, on s'aperçoit que le pseudomorphisme est indispensable pour juger des productions culturelles dans leur diversité. Plutôt que le rejeter, il faut donc faire avec. On remarquera d'ailleurs que dans le langage courant " pseudo " ne signifie plus " tromper " ( $\psi \varepsilon v ́ \delta \omega)$, mais simplement nom d'emprunt, variante, jeu sur les identités.

J'appelle donc pseudomorphisme les effets de ressemblance entre des objets produits dans des contextes culturels différents. Qu'est-ce que cela implique au niveau de l'histoire de l'art ? Tout d'abord que les œuvres n'ont pas de signification propre, qu'elles ont besoin d'être couplées à d'autres qui simultanément les reflètent et les rejettent. La deuxième conséquence est que le pseudomorphisme défiant par définition les sens 
(les choses, de fait, se ressemblent), sa démonstration ne saurait être implicite. Elle doit être manifeste, ouverte, si possible vécue. Il faut apprécier ensemble l'expérience du similaire et du dissemblant, du commun et du divers.

Reprenons Gutai en examinant pour commencer les effets de ressemblance. L'appartenance des œuvres produites par le groupe au champ de l'« avant-garde » (jp. zen'ei, avangyarudo) n'a jamais été sérieusement mise en doute. Dès le premier numéro de la revue Gutai, cette catégorie est utilisée par Yoshihara Jirōo ${ }^{31}$. Spontanément, Michel Tapié en France, comme Dore Ashton aux États-Unis, la reprennent ${ }^{32}$. De même, les œuvres de Gutai sont aujourd'hui partout classées parmi les collections " contemporaines ", que ce soit dans les musées japonais ou dans les musées occidentaux, et les peintres qu'on associe au mouvement sont peu ou prou les mêmes : Jackson Pollock, Georges Mathieu, Lucio Fontana, Sam Francis, Jasper Johns..., liens qui paraissent d'autant plus évidents que les artistes se sont parfois rencontrés. Il existe donc à travers le monde le sentiment que Gutai appartient à un réseau à la fois conceptuel et formel relativement bien identifié. Que l'on se place sur le plan de la chronologie (les années 1950-1960), du cadre existentiel (l'après-guerre), des références artistiques (les avant-gardes, le surréalisme, l'informel, la performance), des matériaux utilisés ou des échanges humains, les facteurs de convergence sont nombreux. Pour la première fois un mouvement « japonais » semble pouvoir participer pleinement d'une avant-garde "internationale ».

Il existe cependant de nombreux facteurs qui empêchent une pleine assimilation de Gutai à l'informel ou à l'expressionnisme abstrait, à commencer par l'histoire dans laquelle s'inscrit le mouvement japonais. Avant la création de Gutai dans les années 1950, aucun artiste est-asiatique n'a été reconnu en Occident comme participant pleinement des avant-gardes et de la "dynamique du progrès ». Seul Fujita Tsuguharu a bénéficié d'une notoriété importante avant-guerre, mais son cas est difficilement comparable, car son succès est indissociable de son installation en France et de son appartenance à l'École de Paris. L'impression d'une incompatibilité des mondes l'emportait encore largement sur toute autre considération. Pourtant, de Kuroda Seiki, qui a séjourné neuf ans en France entre 1884 et 1893, à Okamoto Tarō qui, dans les années 1930, a fréquenté Georges Bataille et son cercle, en passant par Takeuchi Seihō qui visita l'Europe en 1900-1901, de nombreux peintres japonais de la fin du XIX $\mathrm{X}^{\mathrm{e}}$, et du début du XX $\mathrm{X}^{\mathrm{e}}$ siècle, ont développé des techniques et noué des liens en Occident dans l'espoir que, à travers eux, la création de leur pays soit reconnue à parité avec les productions européennes. Mais tous ont été rejetés ou marginalisés, soit parce qu'ils étaient perçus comme des épigones, soit parce que leurs références paraissaient trop lointaines. L'émergence de Gutai sur la scène internationale s'inscrit donc dans un contexte où, d'une part, le sentiment d'altérité radicale était encore très puissant et où, de l'autre, les interconnexions étaient nombreuses, mais nettement dissymétriques et donc porteuses d'intérêts divergents.

Cette différence, importante en soi, paraît encore plus significative quand on la rapporte à la question des pratiques. En Asie de l'Est, l'usage d'encres et de pigments secs favorise comme on le sait le geste sûr, car les repentirs sont difficiles. Dans la tradition européenne de la peinture à l'huile en revanche, la correction et la reprise sont inhérentes au travail plastique. Bien que les œuvres japonaises et occidentales se ressemblent, les histoires dans lesquelles elles s'inscrivent les distinguent fortement. Dans les cas de Pollock et de Mathieu, l'œuvre naît d'une rencontre directe entre la matière et le regard, par-delà la figuration. C'est dans cette rupture avec le réel (la figure) par le réel (la matière), autrement dit dans la resémantisation du réel, que se loge la nouveauté de leur travail. Dans le cas de Gutai, le mouvement est d'un autre ordre. La nouveauté n'est pas dans le geste, l'action ou la vitesse, ni dans le fonctionnement collectif, courant depuis les années $1910^{33}$, mais bien 


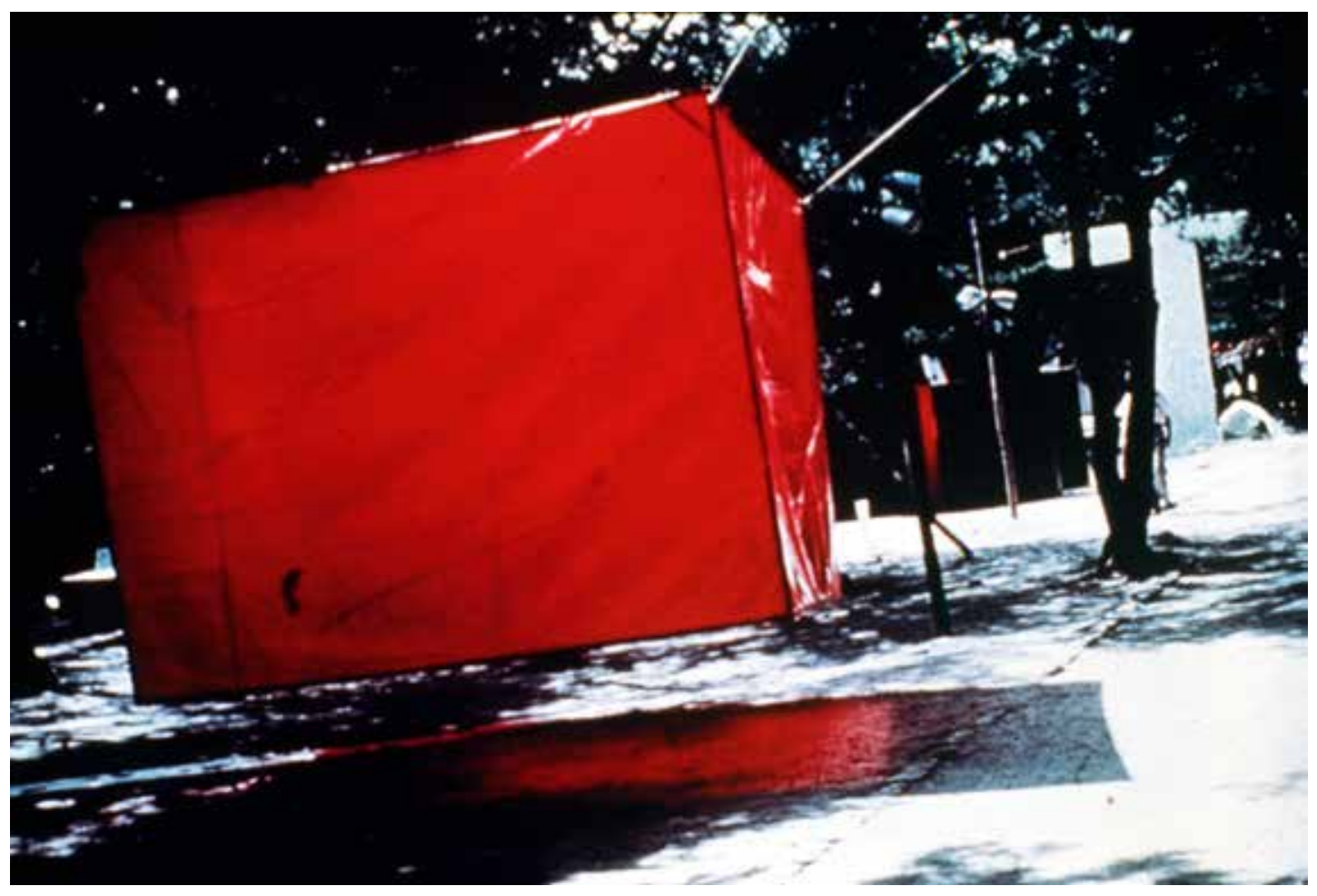

davantage dans l'utilisation de la machine et de matériaux industriels, ainsi que dans la redéfinition de la spatialité des objets et de l'art en général.

3. Yamazaki Tsuruko, Rouge (en forme de moustiquaire), 1956, vinyle, bois, structure métallique, fils électriques, boulons et ampoules, vue de l'installation, Exposition d'art Gutai en plein air, parc Ashiya, sur les berges de la rivière Ashiya, 1956.

Développons un exemple. On a souvent affirmé que le début des années 1960 correspond pour Gutai à un retour au tableau, au détriment des performances. Mais rares sont ceux qui ont remarqué que cette évolution s'accompagne d'un changement dans le mode d'accrochage. Dans les premières expositions du groupe, les tableaux sont quasiment toujours alignés par le bas à un mètre du sol, voire moins. Mais à partir de 1961-1962 la disposition change. Bien que la ligne moyenne reste très basse, l'alignement (qui est rarement tout à fait symétrique) se fait à hauteur du regard. Parallèlement, les murs deviennent blancs, alors qu'au préalable ils pouvaient être ocre, rouges ou bleus. Cette transformation peut s'interpréter comme l'abandon d'un type d'accrochage " traditionnel ", pour suivre le goût occidental et se rapprocher du modèle émergeant du white cube. De fait, l'observation des pratiques américaines et européennes a certainement joué un rôle transformant. Toutefois, la probabilité de l'imitation n'épuise pas le sens de cette évolution, loin de là. L'alignement par le bas est au Japon une pratique ancienne. Très courant au début du $\mathrm{XX}^{\mathrm{e}}$ siècle dans le cadre domestique, il permettait de placer côte à côte en position haute toutes les images protectrices du foyer (à commencer par le portrait des parents). Mais dans le cas des premières expositions Gutai, comme au salon des Indépendants du Yomiuri (Yomiuri andepandan-ten) qui rassemblait alors les avant-gardes, les œuvres étaient au contraire placées près du sol. On était donc déjà passé d'une image " tutélaire " à une image " citoyenne ", chaque œuvre devant être regardée comme un sujet distinct, 
dans une égalité objective de traitement. L'évolution vers un mode d'exposition " américain ", avec l'adaptation de la disposition des images au regard d'un spectateur moyen, marque donc l'abandon de cette conception personnaliste de l'art, pour quelque chose de plus organique, le tassement des œuvres neutralisant par ailleurs le sentiment d'épiphanie recherché dans le cadre du white cube.

Vu sous cet angle, Gutai est bien porteur d'un projet propre, d'une histoire autonome. Le caractère positif des apports de Gutai apparaît aussi très nettement lorsqu'on compare les tentatives du groupe et certaines pratiques contemporaines. L'articulation directe $\mathrm{du}$ local et du global (sans la médiation des structures nationales) ; l'utilisation de l'abstraction comme outil de dépassement des différences socioculturelles ; la conception de l'exposition comme espace de jeu, sont autant de thèmes majeurs du groupe d'Ashiya qu'on retrouve actuellement au Japon avec une vigueur toute particulière ${ }^{34}$. Il y a là une histoire en grande partie endogène. On comprend par la même occasion que Sawaragi, pour qui Gutai se résume à l'action immédiate et violente, ne regarde peut-être pas au bon endroit : au fond, lui-même n'aurait-il pas intériorisé le point de vue occidental ?

Si l'on accepte ce raisonnement, il conviendra de revoir l'échelle des valeurs entre les différents artistes du groupe. L'importance de certaines œuvres pourrait en être rehaussée. Sans doute est-ce le cas de celles qui montrent un nouveau rapport à l'espace, à l'instar des bâches emplies d'eau colorée de Motonaga Sadamasa. Ou encore de toutes celles qui jouent avec l'électricité, comme en ont réalisé Yoshihara Jirō, Tanaka Atsuko et Kanayama Akira, voire avec les propriétés chimiques des matériaux, comme parfois chez Yamazaki Tsuruko et Yoshihara Michio. Plus généralement, il s'agira de remettre en avant les installations, au détriment des performances (fig. 3). Mais l'idée qu'il existe une relation pseudomorphique entre les arts japonais et occidentaux devrait aussi conduire à un regard nouveau sur l'ensemble de l'art " moderne " japonais. Les toiles " réalistes " (shajitsuteki) de Kishida Ryūsei (1891-1929) par exemple ne peuvent pas être correctement jugées selon la seule perspective de l'histoire européenne, pas plus qu'elles ne peuvent l'être selon la seule perspective des traditions locales (fig. 4). Elles témoignent certes de l'utilisation de techniques, de sujets et de modes de composition qui les apparentent formellement à la peinture de la Renaissance italienne et allemande, mais leur sens est incompréhensible à l'aune du réalisme occidental - tout comme l'évolution de la statuaire africaine n'est pas le bon critère pour juger de la période nègre de Picasso. Ce n'est qu'en les resituant dans une histoire à la fois locale et en tension avec l'Occident que l'on pourra retrouver la fraîcheur, l'humour et la profondeur que le public japonais leur reconnaît depuis des décennies.

\section{De l'intérêt comme critère de jugement}

Essayons à présent de rapporter ce que nous avons observé sur les plans historique et formel à une réflexion plus théorique sur l'art et, plus précisément, sur les possibilités de l'esthétique dans le monde à la fois globalisé et fortement compétitif qui est le nôtre aujourd'hui. Parmi tous les courants de pensée contemporains, l'approche contextuelle est de loin celle qui permet le mieux de rendre compte des phénomènes observés plus haut. Dans une conception contextualiste, " les œuvres d'art, comme l'écrit Jerrold Jevinson, sont essentiellement des objets incorporés dans l'histoire ; elles n'ont jamais un statut d'art, des propriétés esthétiques manifestes, des significations artistiques définies, une identité ontologique déterminée, en dehors ou indépendamment de ce contexte génétique déterminé $e^{35}$ ". L'importance cruciale du cadre national dans la réception de Gutai trouve 
ici une explication convaincante. De même que le phénomène de pseudomorphisme, qui veut que des œuvres contemporaines et similaires au niveau formel puissent avoir des significations fortement divergentes. " $\mathrm{Si}$ le contextualisme est correct, des objets partageant une forme manifeste peuvent en réalité n'avoir pas, en tant qu'œuvres d'art, le même statut, le même contenu et la même valeur artistiques ", observe Jevinson ${ }^{36}$.

Toutefois, le contextualisme doit lui-même être élargi. Ce qu'on appelle contexte ne saurait se réduire à des conditions d'énonciation dans un cadre statique et fermé, comme il arrive souvent. Les formes s'inscrivent en effet dans des trajectoires qui n'ont elles-mêmes de sens que par rapport à d'autres trajectoires dans d'autres contextes avec lesquelles elles sont en relation. L'un des points les plus saillants quand on s'intéresse à l'art japonais $\mathrm{du} \mathrm{XX}^{\mathrm{e}}$ siècle tient au constat qu'au niveau international l'intelligibilité, voire même la visibilité des œuvres sont fondamentalement conditionnées par des rapports de force. La réception de Gutai aux États-Unis en est un

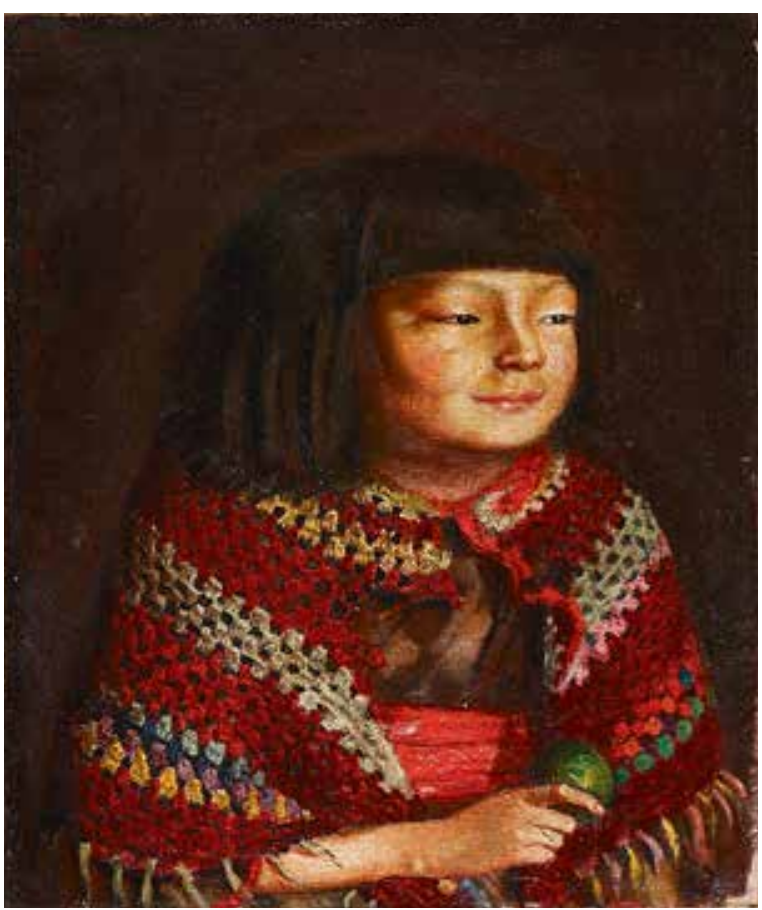

4. Kishida Ryūsei, Reiko souriant, huile sur toile, 45,5 × 38 cm, 1921, Tōkyō, Musée national de Tōkyō, bien culturel important. exemple frappant. L'installation des œuvres, les dispositifs des performances, l'énergie qui s'en dégage, les couleurs et les matériaux choisis ont pu toucher ponctuellement tel ou tel critique, collectionneur ou spectateur, mais l'intérêt porté par ces individus a buté sur un mur pendant des décennies. Jusqu'aux années 2000, Gutai est resté comme invisible à la communauté artistique. De même, des peintres comme Takeuchi Seihō, Kishida Ryūsei ou Okamoto Tarō ont beau avoir une place fondamentale dans l'histoire de l'art japonaise et attirer un public nombreux à chaque exposition, ils restent dans l'ombre en Occident, dans une zone antérieure à toute interprétation. À l'instar de langues lointaines qui peuvent donner à des oreilles non préparées une impression de magma sonore, leurs œuvres semblent prises dans une grisaille indescriptible. Quelque chose qui échappe tant au jugement de goût, qu'à la puissance intrinsèque des formes, précède toute possibilité d'appréciation partagée. Ce qui nous conduit à une première proposition : une approche aréale et polycentrée rend caduque l'idée que l'histoire de l'art procède avant tout d'une série de rencontres entre l'œuvre et le critique. Ce qui domine au contraire est le sentiment que la condition première de l'esthétique est un intérêt collectif pour des systèmes d'œuvres.

De ce postulat découle un second renversement. La séquence interprétation $\rightarrow$ expérience $\rightarrow$ valorisation qui caractérise la conception dominante de l'art moderne, où non seulement ce n'est qu'après un travail d'analyse des divers éléments plastiques et narratifs de l'œuvre que l'expérience peut se produire dans sa forme aboutie, mais où seule une multitude d'expériences permet à la valeur de prendre une dimension véritablement collective, s'inverse lorsqu'on analyse les cultures en tension les unes avec les autres et qu'on sort d'une conception internaliste de l'esthétique qui tend à ne considérer que 
ce qui, dans un cadre donné, a déjà été reconnu comme art. Dans un système pluriel, ce qui prime est l'intérêt, suivi de l'expérience et de l'interprétation. L'intérêt doit ici être compris dans un sens avant tout politique. Pour Tapié et tous ceux qui gravitaient autour de lui (artistes, critiques, marchands, collectionneurs), l'ouverture sur Gutai était une manière de rejouer sur un mode expansif l'histoire de Paris capitale mondiale des arts, de renforcer donc l'autorité de la scène artistique française tout en trouvant des appuis pour contrer la puissance américaine. Ou pour le dire sous la forme d'un jeu de mots, l'intérêt qu'ont éprouvé les partisans de l'informel pour Gutai a d'abord été intéressé. Ce processus semble solidement établi, bien que l'on puisse le regretter et souhaiter qu'il ne reflète qu'un état transitoire de la dynamique des cultures. Le mettre en évidence n'a en effet pas pour objectif d'affirmer la prévalence des approches " réalistes ", au sens des relations internationales, mais de contrer les effets délétères de son occultation.

Prendre acte du caractère intéressé des circulations artistiques est une condition première pour rompre avec l'enfermement dans des regards autocentrés. Car ce travail implique d'intégrer la perspective de l'autre. Sa finalité n'est pas étroitement politique, au sens de la défense des intérêts immédiats d'une communauté, mais de nature éthique. En effet, lorsque les choses sont faites sérieusement (et c'est là toute la différence avec le point de vue réaliste des faucons de la diplomatie), la compréhension en profondeur des trajectoires dans lesquelles se manifeste telle ou telle forme ou énoncé " étranger " implique un effort (séjour de terrain, apprentissage linguistique, lectures, discussions...) qui transforme le sujet de l'intérieur, lui fournissant ce faisant l'occasion d'une véritable expérience. Ce n'est qu'à partir de là que les formes peuvent être interprétées, en commençant de préférence par des logiques d'ensemble. Il en résulte un déplacement assez significatif des usages critiques et muséologiques : non seulement les récits nationaux, les monographies individuelles et les approches non comparatistes en général n'ont guère de pertinence pour rendre compte de ces dynamiques, mais cela implique de franchir encore une étape supplémentaire, à savoir passer d'une histoire de l'art qui prend en charge des chronologies, comme on commence à le voir, à des histoires de l'art (ou des arts, suivant les cas) qui se retrouvent (ponctuellement) pour une topologie (un livre, une exposition, un ensemble coordonné de manifestations...).

Dans la perspective du pseudomorphisme, les emprunts extérieurs sont indissociables de trajectoires internes et les formes qui en émanent n'ont ni le même sens, ni la même valeur que dans le modèle référent. On ne peut pas comparer les formes sans prendre en compte les trajectoires locales. Mais on ne peut pas non plus comprendre les trajectoires locales sans s'intéresser aux interactions avec l'Ailleurs et les conséquences que celles-ci ont sur les formes. Autrement dit, l'art " moderne " ou " contemporain " doit être montré en tension : en tension avec ce qui formellement ressemble, mais s'oppose historiquement. En tension avec ce qui formellement s'oppose, mais ressemble structurellement. Voilà la manière dont j'aimerais voir les œuvres présentées dans nos musées - mais il s'agit là en fait d'une position qu'on pourrait élargir à toute l'écriture de l'histoire : c'est-à-dire non pas de façon nationale ou régionale, ni même globale ou connectée, mais contentieuse et différentielle, dans une pluralité active donc, qui donne à voir l'enchevêtrement tourbillonnant de trajectoires qui se rapprochent et se repoussent. 


\section{Michael Lucken}

Michael Lucken est professeur à l'INALCO (Paris). Historien et historien de l'art, il est spécialiste du Japon moderne. Après s'être intéressé aux représentations de la Seconde Guerre mondiale en Asie de l'Est, il étudie depuis une dizaine d'années les mécanismes de circulation et d'appropriation des savoirs et des formes. Il a publié, entre autres, L'Art du Japon au vingtième siècle (Paris, Hermann, 2001) ; 1945, Hiroshima : les imagessources (Paris, Hermann, 2008) ; Les Japonais et la guerre, 1937-1952 (Paris, Fayard, 2013) ; Nakai Masakazu. Naissance de la théorie critique au Japon (Dijon, Les presses du réel, 2015) ; Imitation and Creativity in Japanese Arts. From Kishida Ryūsei to Miyazaki Hayao (New York, Columbia University Press, 2016) ; Le Japon grec. Culture et possession (Paris, Gallimard, 2019).

\section{NOTES}

1. Hariu Ichirō, "Jihyōteki bijutsu-ron : Gutai-ten " [Critique d'art actuel : I'exposition Gutai], dans Mizue, $n^{\circ}$ 628, novembre 1957. En français, voir notre traduction dans Antoni Tàpies, Michael Lucken, Éric Mézil et al., Gutai, cat. exp. (Paris, Galerie nationale du Jeu de Paume, 1999), Paris, éditions du Jeu de Paume, 1999, p. 215. Nota bene : sauf mention contraire, toutes les traductions du japonais et de l'anglais sont les miennes.

2. Reiko Tomii, Radicalism in the Wilderness: International Contemporaneity and the 1960s Art in Japan, Cambridge, MIT Press, 2016, p. 12.

3. Alfred Pacquement, "Gutai : I'extraordinaire intuition ", dans Le Japon des avant-gardes, cat. exp. (Paris, Centre Georges Pompidou, 1987), Paris, éditions du Centre Pompidou, 1986 ; Laurence Bertrand Dorléac, L'Ordre sauvage : violence, dépense et sacré dans l'art des années 1950-1960, Paris, Gallimard, 2004 ; Marc Dachy, Dada au Japon, Paris, PUF, 2002. Parmi les publications récentes, citons Florence de Mérédieu, «Défi au soleil de la mi-été », dans Gutai : l'espace et le temps, cat. exp. (Rodez, musée Soulages, 2018), Rodez, musée Soulages, 2018 ; Astrid Handa-Gagnard, "I Think I Want the Courage to Give up Painting ", dans Minoru Onoda, Zurich, Scheidegger \& Spiess, 2019, p. 19-27.

4. Sur le débat autour de Tapié, voir, entre autres, Éric Mézil, " "Nul n'est prophète en son pays" : le cas de Michel Tapié ", dans Tàpies, Lucken, Mézil, 1999, cité n. 1, p. 25-41 ; Juliette Évezard, "Michel Tapié : I'invité de Gutai ", dans Gutai : I'espace et le temps, 2018, cité n. 3, p. 36-41.

5. Alexandra Munroe, "All the Landscapes: Gutai's World ", dans Ming Tiampo, Alexandra Munroe (dir.), Gutai: Splendid Playground, cat. exp. (New York, The Solomon R. Guggenheim Museum), New York, Guggenheim Foundation, 2013, p. 29.

6. Voir Dore Ashton, "Japanese Avantgardism ", dans Arts \& Architecture, $\mathrm{n}^{\circ} 75$, novembre 1958, reproduit dans Gutai shiryō-shū. Dokyumento Gutai, 1954-1972
[Documents Gutai, 1954-1972], Ashiya, Ashiya-shi bunka shinkō zaidan, 1993, p. 322-323.

7. Michael Kirby, «Introduction», dans Happenings: an Illustrated Anthology, New York, Dutton, 1965, p. 28-29.

8. Voir notamment Joan Kee, "Situating a Singular Kind of 'Action': Early Gutai Painting, 1954-1957 ", dans Oxford Art Journal, vol. 26, n² 2, 2003 ; Midori Yoshimoto, Into Performance: Japanese Women Artists in New York, New Brunswick, Rutgers University Press, 2005 ; Ming Tiampo, Gutai - Decentering Modernism, Chicago, Chicago University Press, 2011 ; Ming Tiampo, "Gutai Chain: the Collective Spirit of Individualism », dans Reiko Tomii et Midori Yoshimoto (dir.), Collectivism in 20th-Century Japanese Art, numéro de Positions: Asia critique, vol. 21, $\mathrm{n}^{\circ} 2$ 2, 2013, p. 383-415; Gabriel Ritter (dir.), Between Action and the Unknown: the Art of Kazuo Shiraga and Sadamasa Motonaga, New Haven, Yale University Press, 2015 ; Namiko Kunimoto, The Stakes of Exposure: Anxious Bodies in Postwar Japanese Art, Minneapolis, University of Minnesota Press, 2017.

9. Kawasaki Kōichi (1952-2019) fut longtemps le directeur du musée municipal d'Ashiya (où est conservée une partie des archives de Gutai), avant de devenir professeur à l'Université de jeunes filles de Kōnan. Osaki Shin'ichirō (né en 1962) est conservateur en chef au musée départemental de Tottori ; voir notamment Osaki Shin'ichirō, "Gutai bijutsu kyōkai wa ika ni rekishika sareta ka » [Comment l'Association des beaux-arts Gutai a-t-elle été inscrite dans I'histoire ?], dans Bijutsu fōramu, n 21, vol. 30, 2014. Hirai Shōichi (né en 1962) a été conservateur dans plusieurs musées départementaux et nationaux, avant d'être nommé en 2012 professeur d'histoire de l'art à l'université du Kansai.

10. Ming Tiampo, "Please Draw Freely », dans Tiampo, Munroe, 2013, cité n. 5, p. 75.

11. Voir, entre autres, Michael Lucken, L'Art du Japon au vingtième siècle. Pensées, formes, résistances, Paris, Hermann, 2001, p. 182 ; Bertrand Dorléac, 2004, cité n. 3, p. 48.

12. Okabe Aomi, "Action et avant-garde", dans Le Japon des avant-gardes, 1986, cité n. 3, p. 350.

13. Itō Junji, "Gutai ou l'énigme du Japon », dans Tàpies, Lucken, Mézil, 1999, cité n. 1, p. 167-177.

14. Hirai Shōichi, "Kansai ni okeru zen.ei bijutsu no kenkyū: Gutai bijutsu kyōkai wo chūshin ni » [Étude sur les arts d'avant-garde dans le Kansai : autour de I'Association artistique Gutai], thèse de doctorat, université Kansai daigaku, mars 2006.

15. Tan'o Yasunori (né en 1950), professeur à l'université Waseda de 1986 à 2020 ; Omuka Toshiharu (né en 1951), professeur à l'université de Tsukuba de 1992 à 2017 ; Kinoshita Naoyuki (né en 1954), professeur à l'université de Tōkyō de 2000 à 2017 ; Ushiroshōji Masahiro (né en 1954), professeur à l'université de Kyūshū de 2002 à 2018 ; Satō Dōshin (né en 1956), professeur à l'université des Arts de Tōkyō depuis 1994 ; Inaga Shigemi (né en 1957), professeur au Centre de recherche international sur la culture japonaise ou Nichibunken depuis 1997. Ensemble, ces enseignants-chercheurs constituent le noyau dur de la formation doctorale en histoire de l'art moderne et contemporain au Japon depuis les années 1990. 
16. Voir Kitazawa Noriaki, "Nihonga" no ten'i [Les basculements du "nihonga"], Tōkyō, Buryukke, 2003, p. 91, 175 ; Inaga Shigemi, Nihon bijutsushi no kindai to sono gaibu [La modernité de l'histoire de l'art japonais et ses frontières], Tōkyō, Hōsō daigaku kyōiku shinkōkai, 2018, p. 187. Citons aussi le cas du livre d'Adachi Gen, Zen'ei no idenshi: anakizumu kara sengo bijutsu e [Les gênes de l'avant-garde : de l'anarchisme à l'art d'après-guerre], Tōkyō, Buryukke, 2012, où Gutai n'est même pas mentionné.

17. Notons plusieurs travaux récents de qualité qui privilégient l'approche individuelle à l'étude de groupe : Ikegami Hiroko, « Kōi toshite no aburae : Shiraga Kazuo no Doro ni idomu » [La peinture à I'huile comme action : Défi à la boue de Shiraga Kazuo], dans Ninagawa Junko (dir.), Yusai e no shōdō [L'appel de l'huile], Tōkyō, Chūō kōron bijutsu shuppan, 2015 ; Katō Mizuho, "Kanayama Akira no dendōkiki ni yoru byōga: Gutai bijutsu kyōkai ni okeru sono igi " [Les dessins à la machine à propulsion électrique de Kanayama Akira : leur sens dans le cadre de l'Association des beauxarts Gutai], dans Bijutsushi, n 67-1, 2017, p. 1-16 ; et Matsumi Teruhiko, "Shimamoto Shōzō no geijutsu kyōiku katsudō ni tsuite: āru buryutto no kanten kara no apurōchī » [Le travail d'enseignement artistique de Shimamoto Shōzō : un regard du point de vue de I'Art brut], dans Bijutsu kyōikugaku kenkyū, no 49-1, 2017, p. 377-384.

18. Voir Nakajima Izumi, Anchi akushon : Nihon sengo bijutsu to josei gaka [Anti-action : l'art japonais d'aprèsguerre et les femmes peintres], Tōkyō, Buryukke, 2019.

19. Kuroda Raiji, Nikutai no anākizumu: 1960 nendaiNihon bijutsu ni okeru pafōmansu no chika suimyaku [L'anarchie des corps : les années 1960 - courants souterrains de la performance dans l'art japonais], Tōkyō, Guramubukkusu, 2010, p. 529, 530. Pour la présentation du groupe, voir p. 109-116.

20. Nakazawa Hideki, Nihon gendai-shi: Nihon-hen 1945-2014 [Histoire de l'art contemporain : Japon, 19452014], Tōkyō, Āto daibā, 2014, p. 28.

21. Sawaragi Noi (né en 1962) s'est fait connaître comme critique d'art dans les années 1990. Il est nommé maître de conférences à I'Université des beaux-arts de Tama en 2000 où il enseigne comme professeur depuis 2010.

22. Sawaragi Noi, Nihon / Gendai / Bijutsu [Art / Japon / Contemporain], Tōkyō, Shinchōsha, 1998, p. 8-14. Toute l'introduction du livre de Sawaragi est une discussion sur Gutai qui s'appuie, en les contestant, sur deux textes importants des années 1970-1980, I'un de Hikosaka Naoyuki (1973) qui défend l'idée que Gutai a permis de sortir de la domination occidentale et marque un tournant dans l'art d'après-guerre, l'autre de Chiba Shigeo (1986) qui souligne que la posture de Gutai - le choix de la praxis (jp. purakushisu) au détriment de la poièsis (jp. poiēshisu) - se retrouve dans d'autres mouvements tout au long des années 1960. Voir Hikosaka Naoyuki, "Tojirareta enkan no kanata wa... Gutai no kiseki kara nani wo... » [De l'autre côté du cercle fermé... Où mènent les traces de Gutai ?], dans Bijutsu techō, no 380, août 1973 ; Chiba Shigeo, Gendai bijutsu itsudatsu-shi 1945-1985 [Histoire détournée de l'art d'après-guerre 1945-1985], Tōkyō, Shōbun-sha, 1986.
23. Sawaragi, 1998, cité n. 22, p. 10, 11.

24. Ibidem, p. 12.

25. Notons qu'il existe cependant quelques artistes se plaçant ouvertement dans la filiation de Gutai, comme par exemple le peintre Nakamura Jōji (né en 1974).

26. Béatrice Joyeux-Prunel, Les Avant-gardes artistiques. Une histoire transnationale, 2 vol., Paris, Folio, 20162017.

27. Georges Didi-Huberman, Devant le temps, Paris, éditions de Minuit, 2000, p. 20.

28. Andrea Pinotti, «Chi ha paura dello pseudomorfo? ", dans Rivista di estetica, $\mathrm{n}^{\circ}$ 62, septembre 2016 p. 89-95.

29. Didi-Huberman, 2000, cité n. 27, p. 16.

30. Ibidem, p. 11.

31. Yoshihara Jirō, " Hakkan ni sai shite » [À I'occasion du lancement de la publication], reproduit dans Gutai shiryō-shū..., 1993, cité n. 6, p. 268.

32. Michel Tapié, préface, dans Michel Tapié et Haga Tōru, Continuité et avant-garde au Japon, Turin, Fratelli Pozzo, 1961, passim, n. p. ; Ashton, (1955) 1993, cité n. 6, p. 322

33. Voir Lucken, 2001, cité n. 11, p. 44-50.

34. Voir par exemple les installations Chūshō-Kazoku [Abstraction - Famille] de Tanaka Kōki, et Āto pureguraundo [Terrain de jeu artistique], d'Endō Mikiko et Hibino Katsuhiko, lors de la Triennale d'Aichi, au musée départemental d'Aichi, Nagoya, $1^{\text {er }}$ août - 14 octobre 2019; ou encore l'exposition Asobi no jikan [Le temps du jeu], au musée d'Art contemporain de la Ville de Tōkyō (MOT), 20 juillet - 10 octobre 2019.

35. Jerrold Jevinson, "Le contextualisme esthétique », R. Pouivet (trad. fra.), dans Esthétique contemporaine. Art, représentation et fiction, Jean-Pierre Cometti, Jacques Morizot et Roger Pouivet (éd.), Paris, Vrin 2005, p. 452.

36. Ibidem. 